Verhältnis zwischen der Teilchenzahl in Meereshöhe und der Zahl der Primärteilchen angibt, beides für senkrechten Einfall, da die erhöhte Absorption für schrägen Einfall schon durch $\cos ^{2} \alpha$ berücksichtigt ist.

Strahlt dagegen die Sonne im zeitlichen und räumlichen Durchschnitt pro $\mathrm{cm}^{2}$ und $\sec N_{0, \text { sol }}$ Teilchen in die Raumwinkeleinheit ab, so würde eine im Abstand der Erde gegen die Sonne gerichtete gleiche Apparatur bei Abschirmung durch die Atmosphäre

$$
N_{\mathrm{sol}}=P F \pi\left(\frac{R}{E}\right)^{2} N_{0, \mathrm{sol}}=N_{0, \mathrm{sol}} P F / 15000
$$

Teilchen registrieren. Dabei ist $R$ der Sonnenradius $\approx 7 \cdot 10^{10} \mathrm{~cm}$ und $E$ die mittlere Entfernung von der Sonne $\approx 1,5 \cdot 10^{13} \mathrm{~cm}$. Weiter ist impliziert, daß die Sonne die Ultrastrahlung nach allen Richtungen im zeitlichen Mittel in gleicher Stärke aussendet.

Mit dem oben abgeschätzten Mittelwert $N_{\text {sol }}$ $=0,03 \cdot N_{\text {allg. }}$ folgt aus (1) und (2)
$N_{0, \text { sol }}=450 N_{0, \text { allg. }}$.

D.h. bei unseren Voraussetzungen, daß die Sonne mindestens 450-mal soviel Ultrastrahlungsteilchen mit magnetischen Steifigkeiten $\geqq 1,2 \cdot 10^{7} \mathrm{Gauß} \cdot \mathrm{cm}$ ausgestrahlt hat, als sie maximal absorbieren konnte. Diese Zahl basiert nur auf den verhältnismäßig seltenen größeren Effekten. Es erscheint nicht ausgeschlossen, daß eine größere Anzahl kleiner und deshalb nicht erfaßter Effekte einen noch größeren Beitrag zu $N_{0 \text {, sol }}$ liefern würde.

Beim sichtbaren Licht ist das Verhältnis $J_{0, \text { sol }} / J_{0, \text { Fixsterne }}$ um mehrere Größenordnungen größer. Nimmt man also an, daß die Ultrastrahlungsemission bei anderen Sternen ähnlicher Spektraltypen in ähnlichem Verhältnis wie bei der Sonne erfolgt, so reicht diese Ultrastrahlung bei geradliniger Ausbreitung keineswegs aus, um die Intensität der allgemeinen Ultrastrahlung zu erklären. Da aber die Ultrastrahlung ablenkbar ist, kann sie in sehr ausgedehnten und zeitlich konstanten Magnetfeldern, wie z. B. in einem heute öfters diskutierten galaktischen Magnetfeld, selbst bei geringster Feldstärke festgehalten und sogar im Lauf der Zeit angesammelt werden, weil die Absorption infolge der geringen Raumerfüllung mit Materie nur sehr langsam erfolgt. Ob damit die allgemeine Ultrastrahlung zum größeren Teil auf ähnliche Vorgänge wie die solare Ultrastrahlung zurückgeführt werden kann, läßt sich bei den heutigen unsicheren Unterlagen keineswegs entscheiden. Immerhin ist mit dieser Möglichkeit zu rechnen.

Die vorliegende Arbeit wurde in der For schung sstelle für Physik der Stratosphäre der Kaiser-Wilhelm-Gesellschaft ausgeführt. Dem Leiter der Forschungsstelle, Hrn. Prof. Dr. E. Regener, danke ich für die stetige großzügige Unterstützung dieser auf längere Sicht angelegten Arbeiten herzlichst.

\title{
Einige allgemeine Beziehungen für Zustandsfunktionen bei Vielkomponentensystemen
}

\author{
Von Rolf HaAse \\ Aus dem Physikalisch-chemischen Institut der Universität Marburg \\ (Z. Naturforschg. 3 a, 285-290 [1948]; eingegangen am 23. Februar 1948)
}

\begin{abstract}
Aus allgemeingültigen thermodynamischen Beziehungen wird ein Zusammenhang zwischen partiellen molaren und mittleren molaren Zustandsgrößen hergeleitet. Aus diesem Zusammenhang folgen u. a. die Grundlagen der bekannten geometrischen Verfahren zur Ermittlung partieller molarer Größen bei Zwei- und Dreistoffgemischen. Ferner ergeben sich Rechenvereinfachungen für die analytische Bestimmung der partiellen molaren Zustandsfunktionen bei Systemen mit beliebig vielen Komponenten. Die Koexistenzbedingungen für zwei Phasen werden in eine solche Form gebracht, daß die Grundgleichungen der vielbenutzten geometrischen Darstellungen ebenfalls als Spezialfälle der allgemeinen Gleichungen für beliebig viele Komponenten erkannt werden. Einige Anwendungen der abgeleiteten Beziehungen auf Verdampfungsgleichgewichte werden gebracht.
\end{abstract}

I n der Literatur wird für die Darstellung von partiellen molaren Größen bei binären und ternären Gemischen häufig von geometrischen Ver- anschaulichungen Gebrauch gemacht. Da diese Methode bei Systemen mit mehr als drei Komponenten versagt, erscheint es wünschenswert, den 
allgemeinen analytischen Ausdruck zu finden, der für beliebige Systeme gilt.

Auch der geometrischen Darstellung der Koexistenzbedingungen für Zwei- und Dreistoffsysteme muß eine allgemeine analytische Beziehung zugrunde liegen. Diese soll auf ebenso einfache wie allgemeine Weise abgeleitet werden.

$z$ sei ein beliebiger Extensitätsparameter, d. h. eine thermodynamische Zustandsfunktion, die in bezug auf die Molzahlen aller Komponenten homogen und ersten Grades ist (z. B. Volumen, Energie, Entropie). Wir wählen als unabhängige Variable die Molzahlen $m_{1}, m_{2} \ldots m_{n}$, den Druck $P$ und die Temperatur T. Dann gilt:

$$
d z=\frac{\partial z}{\partial P} d P+\frac{\partial z}{\partial T} d T+\sum_{k=1}^{n} \frac{\partial z}{\partial m_{k}} d m_{k} .
$$

Ferner ist wegen des Eulerschen Satzes:

$$
z=\sum_{k=1}^{n} m_{k} \frac{\partial z}{\partial m_{k}}
$$

Daraus ergibt sich:

$$
d z=\sum_{k=1}^{n} m_{k} d \frac{\partial z}{\partial m_{k}}+\sum_{k=1}^{n} \frac{\partial z}{\partial m_{k}} d m_{k}
$$

Aus (1) und (3) folgt die verallgemeinerte Gibbs-Duh em sche Beziehung ${ }^{1}$ :

$$
\sum_{k=1}^{n} m_{k} d \frac{\partial z}{\partial m_{k}}-\frac{\partial z}{\partial P} d P-\frac{\partial z}{\partial T^{\prime}} d T=0
$$

Wir setzen für eine beliebige Komponente $i$ :

$$
\begin{gathered}
\frac{\partial z}{\partial m_{i}}=z_{i}, \\
\frac{z}{\sum_{k=1}^{n} m_{k}}=Z, \\
\frac{m_{i}}{\sum_{k=1}^{n} m_{k}}=x_{i} .
\end{gathered}
$$

1 E. A. G u g g e n h e i m, Modern Thermodynamics, London 1933, S. 30.
Wir erhalten aus (2), (5), (6) und (7):

$$
Z=\sum_{k=1}^{n} x_{k} z_{k} \text {. }
$$

Aus (4), (5) (6) und (7) ergibt sich:

$$
\sum_{k=1}^{n} x_{k} d z_{k}-\frac{\partial Z}{\partial P} d P-\frac{\partial Z}{\partial T} d T=0 .
$$

$Z$ ist die (mittlere) molare Zustandsgröße der Mischung, $z_{i}$ die partielle molare Größe der Komponente $i$ und $x_{i}$ der Molenbruch der Komponente $i$. Die Molenbrüche sind jedoch nicht alle unabhängig; denn zwischen ihnen besteht die Identität:

$$
\sum_{k=1}^{n} x_{k}=1
$$

Wir wählen als unabhängige Molenbrüche dieder $(n-1)$ ersten Komponenten; dann gilt für den Molenbruch der $n$-ten Komponente:

$$
x_{n}=1-\sum_{k=1}^{n-1} x_{k} .
$$

Daraus erhalten wir:

$$
\frac{\partial x_{n}}{\partial x_{i}}=-1 .
$$

Aus (2a) und (9) folgt:

$$
\frac{\partial Z}{\partial x_{i}}=\sum_{k=1}^{n} x_{k} \frac{\partial z_{k}}{\partial x_{i}}+z_{i}-z_{n}
$$

Da diese Differentiation bei konstantem $P$ und $T$ ausgeführt ist, folgt aus (4a):

$$
\sum_{k=1}^{n} x_{k} \frac{\partial z_{k}}{\partial x_{i}}=0 .
$$

Aus den beiden letzten Gleichungen ergibt sich die wichtige Beziehung:

$$
\frac{\partial Z}{\partial x_{i}}=z_{i}-z_{n} .
$$

Substituieren wir in (2a) $z_{k}$ mit (10), so erhalten wir: 


$$
z_{n}=Z-\sum_{k=1}^{n-1} x_{k} \frac{\partial Z}{\partial x_{k}}
$$

Für $n=2$ folgt:

$$
\begin{aligned}
& z_{2}=Z-x_{1} \frac{\partial Z}{\partial x_{1}} \\
& z_{1}=Z-x_{2} \frac{\partial Z}{\partial x_{2}}
\end{aligned}
$$

Dies führt zu einer bekannten Darstellung der partiellen molaren Größen im $Z$ - $x$-Diagramm ${ }^{1,2}$ : $z_{2}$ ist der durch die Tangente an den betreffenden Punkt der $Z$ - $x$-Kurve auf der Achse $x_{2}=1$ erzeugte Ordinatenabschnitt, $z_{1}$ der entsprechende Abschnitt auf der Achse $x_{1}=1$.

Für $n=3$ ergibt sich aus (11):

$$
\begin{aligned}
& z_{1}=Z-x_{2} \frac{\partial Z}{\partial x_{2}}-x_{3} \frac{\partial Z}{\partial x_{3}}, \\
& z_{2}=Z-x_{1} \frac{\partial Z}{\partial x_{1}}-x_{3} \frac{\partial Z}{\partial x_{3}} \\
& z_{3}=Z-x_{1} \frac{\partial Z}{\partial x_{1}}-x_{2} \frac{\partial Z}{\partial x_{2}} .
\end{aligned}
$$

Hieraus folgt ebenfalls eine einfache Beziehung bei der Darstellung der Funktion $Z$ in einem räumlichen Koordinatensystem, in dem zwei Molenbrüche als Variable in einer Ebene und die $Z$-Werte durch auf dieser Ebene senkrecht errichtete Ordinaten abgebildet sind. Nehmen wir in der Ebene Möbiussche Dreieckskoordinaten (das Gibbssche Darstellungsdreieck), so entsprechen die drei Eckpunkte $A_{1}, A_{2}, A_{3}$ des gleichseitigen Dreiecks den Werten $x_{1}=1, x_{2}=1$, $x_{3}=1$. Die Abschnitte auf den in $\mathrm{A}_{1}, \mathrm{~A}_{2}, \mathrm{~A}_{3}$ errichteten Loten, erzeugt durch die an einen Punkt $\mathrm{P}$ der $Z$-Fläche gelegte Tangentialebene, geben dann die Werte der partiellen molaren Größen $z_{1}, z_{2}, z_{3}$ für den Punkt $\mathrm{P}$ an ${ }^{1}$.

Für $n>3$ ist eine graphische Darstellung nicht möglich. Man muß jetzt analytisch vorgehen und die partiellen molaren Größen nach (11) durch Differentiation ableiten. Da im allgemeinen die mittlere Zustandsfunktion $Z$ in Abhängigkeit von den $x_{k}$ eine für die Durchführung der Differentiation einfachere Gestalt hat als die totale Zustands-

${ }^{2}$ G. N. L e w is u. M. R a nd a ll, Thermodynamik, Übersetzung von O. Redlich, Wien 1927, S. 31 . größe $z$ in Abhängigkeit von den $m_{k}$, ist die Berechnung der partiellen Größen nach (11) der üblichen Methode vorzuziehen. Dies sieht man schon im Falle binärer Gemische. Will man z. B. die partiellen molaren Mischungsentropien eines idealen $\mathrm{Z}$ weistoffgemisches aus der bekannten Beziehung

$$
\Delta S=-R(x \ln x+(1-x) \ln (1-x))
$$

( $R=$ Gaskonstante) gewinnen, so ist die Rechnung nach (12a) und (12b) einfacher als nach der gewöhnlich benutzten Methode, bei der man setzt:

$$
\begin{gathered}
x=\frac{m_{1}}{m_{1}+m_{2}}, \\
\Delta s=\left(m_{1}+m_{2}\right) \Delta S, \\
\Delta s_{i}=\frac{\partial}{\partial m_{i}} \Delta s .
\end{gathered}
$$

Von allen partiellen molaren Zustandsfunktionen hat eine Größe besondere Bedeutung: das von Gibbs eingeführte chemische Potential $\mu_{i}$, definiert durch:

$$
\mu_{i}=\left(\frac{\partial g}{\partial m_{i}}\right)_{T, P, m_{j}}(j \neq i ; j=1,2 \ldots n) .
$$

$g$ ist die totale freie Enthalpie (Gibbssche freie Energie). Das chemische Potential hat in zwei Phasen (gekennzeichnet durch einen bzw. zwei Striche), die miteinander im Gleichgewicht sind, für jede Komponente den gleichen Wert:

$$
\mu_{i}^{\prime}=\mu_{i}^{\prime \prime} .
$$

Wegen dieser fundamentalen Eigenschaft hat G u g genheim ${ }^{3}$ für $\mu_{i}$ an Stelle des Namens ,partielle molare freie Enthalpie“ die Gibbssche Bezeichnung „chemisches Potential“" wieder eingeführt und neben die Definitionsgleichung (14) noch drei andere gleichwertige Gleichungen gesetzt. Im vorliegenden Falle, in dem $P$ und $T$ als unabhängige Variable gewählt sind, interessiert uns aber nur Gl. (14).

Setzen wir für $Z$ die molare freie Enthalpie der Mischung $G$, für $z_{i}$ die Größe $\mu_{i}$, so erhalten wir aus (10) und (15):

$$
\left(\frac{\partial G}{\partial x_{i}}\right)^{\prime}=\left(\frac{\partial G}{\partial x_{i}}\right)^{\prime \prime} .
$$

${ }^{3}$ E. A. Gug g e n h e i m ${ }^{1}$, S. $23,30$. 
Aus (11) und (15) ergibt sich:

$$
\left(G-\sum_{k=1}^{n-1} x_{k} \frac{\partial G}{\partial x_{k}}\right)^{\prime}=\left(G-\sum_{k=1}^{n-1} x_{k} \frac{\partial G}{\partial x_{k}}\right)^{\prime \prime}
$$

Die Gln. (16) und (17) ersetzen in gewissem Sinne die einfachere Koexistenzbeziehung (15), bieten aber bestimmte Vorteile, besonders für graphische Darstellungen von Gleichgewichten bei ternären Systemen.

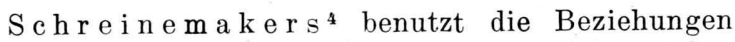
(16) und (17), um über Mehrphasengleichgewichte von Vielkomponentensystemen gewisse Sätze zu gewinnen, gibt aber keine allgemeine Herleitung. Die für $n=3$ gegebene Ableitung ${ }^{5}$ ist umständlich.

Für $n=2$ folgt auss (16) und (17), wenn wir den einzigen unabhängigen Molenbruch $x$ nennen:

$$
\begin{gathered}
\left(\frac{\partial G}{\partial x}\right)^{\prime}=\left(\frac{\partial G}{\partial x}\right)^{\prime \prime} \\
\left(G-x \frac{\partial G}{\partial x}\right)^{\prime}=\left(G-x \frac{\partial G}{\partial x}\right)^{\prime \prime} .
\end{gathered}
$$

Aus diesen zuerst von van der Waals ${ }^{\bullet}$ angegebenen Beziehungen liest man ab: Wenn die $(G, x)$-Kurve eine solche Form hat, daß man eine Doppeltangente in zwei Punkten an diese Kurve legen kann, so stellen die beiden Punkte koexistente Phasen dar. Gl. (18a) verlangt nämlich gleiche Neigung der Tangenten, und Gl. (18b) gleiche Schnittpunkte auf den Ordinatenachsen.

Für $n=3$ ergibt sich:

$$
\begin{gathered}
\left(\frac{\partial G}{\partial x_{1}}\right)^{\prime}=\left(\frac{\partial G}{\partial x_{1}}\right)^{\prime \prime} \\
\left(\frac{\partial G}{\partial x_{2}}\right)^{\prime}=\left(\frac{\partial G}{\partial x_{2}}\right)^{\prime \prime} \\
\left(G-x_{1} \frac{G}{\partial x_{1}}-x_{2} \frac{\partial G}{\partial x_{2}}\right)^{\prime} \\
=\left(G-x_{1} \frac{\partial G}{\partial x_{1}}-x_{2} \frac{\partial G}{\partial x_{2}}\right)^{\prime \prime} .
\end{gathered}
$$

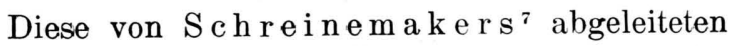
und viel angewendeten Gleichungen besagen: Hat die $G$-Fläche eine solche Form, daß man an zwei Punkte eine Doppelberührungsebene legen kann,

4 H. W. B a khuis R o z e bo o m, Die heterogenen Gleichgewichte, Braunschweig, 3. Heft, 1. Teil (Abhandlung von F. A. H. S c h re in e m a ker s, 1911), S. 282 . so gibt es zwei koexistente Phasen, deren Zusammensetzungen durch diese Punkte angegeben werden. - Schließlich werde noch die Anwendung einiger der abgeleiteten Gleichungen auf ein Spezialproblem gezeigt.

Kehren wir zu Gl. (4 a) zurück und setzen wir:

$$
z_{k}=\mu_{k}, \frac{\partial Z}{\partial P}=\frac{\partial G}{\partial P}=V, \frac{\partial Z}{\partial T}=\frac{\partial G}{\partial T}=-S .
$$

( $V=$ Volumen, $S=$ Entropie). Damit erhalten wir:

$$
\sum_{i=1}^{n} x_{k} d \mu_{k}=V d P-S d T
$$

Diese Gleichung (die Gibbs-Duhemsche Beziehung in der bekannten Form) muß für jede Phase gelten.

Für Gleichgewicht zwischen zwei Phasen gilt:

$$
\begin{aligned}
& d T^{\prime}=d T^{\prime \prime}=d T^{\prime} \\
& d P^{\prime}=d P^{\prime \prime}=d P \\
& d \mu_{k}^{\prime}=d \mu_{k}^{\prime \prime}=d \mu_{k} .
\end{aligned}
$$

Die Koexistenzbedingung für zwei Phasen lautet also:

$$
\begin{aligned}
& \sum_{k=1}^{n} x_{k}^{\prime} d \mu_{k}=V^{\prime} d P-S^{\prime} d T \\
& \sum_{k=1}^{n} x_{k}^{\prime \prime} d \mu_{k}=V^{\prime \prime} d P-S^{\prime \prime} d T .
\end{aligned}
$$

Es ergibt sich ferner aus (10):

$$
\begin{gathered}
\frac{\partial G}{\partial x_{i}}=\mu_{i}-\mu_{n}, \\
d \mu_{i}=d \frac{\partial G}{\partial x_{i}}+d \mu_{n}, \\
\sum_{k=1}^{n-1} x_{k} d \mu_{k}=\sum_{k=1}^{n-1} x_{k} d \frac{\partial G}{\partial x_{k}}+\sum_{k=1}^{n-1} x_{k} d \mu_{n} .
\end{gathered}
$$

Daraus folgt:

$$
\sum_{k=1}^{n} x_{k} d \mu_{k}=\sum_{k=1}^{n-1} x_{k} d \frac{\partial G}{\partial x_{k}}+d \mu_{n} .
$$

${ }^{5} \mathrm{H}$. W. B. R o o z e b o o $\mathrm{m}^{4}$, S. 250.

6 J. D. van der Waals, Z. physik. Chem. 5, 133 [1890].

7 F. A. H. S c h r e in e m a k e r s, Z. physik. Chem. 36, 271 [1901]. 
Wir erhalten also mit $d\left(\frac{\partial G}{\partial x_{k}}\right)^{\prime}=d\left(\frac{\partial G}{\partial x_{k}}\right)^{\prime \prime}=d \frac{\partial G}{\partial x_{k}}$ aus (21):

$\sum_{k=1}^{n-1}\left(x_{k}^{\prime}-x_{k}^{\prime \prime}\right) d \frac{\partial G}{\partial x_{k}}=\left(V^{\prime}-V^{\prime \prime}\right) d P-\left(S^{\prime}-S^{\prime \prime}\right) d T$.

Nun gilt:

$$
\begin{gathered}
\frac{\partial}{\partial P} \frac{\partial G}{\partial x_{k}}=\frac{\partial}{\partial x_{k}} \frac{\partial G}{\partial P}=\frac{\partial V}{\partial x_{k}} \\
\frac{\partial}{\partial T} \frac{\partial G}{\partial x_{k}}=\frac{\partial}{\partial x_{k}} \frac{\partial G}{\partial T}=-\frac{\partial S}{\partial x_{k}} \\
d \frac{\partial G}{\partial x_{k}}=\frac{\partial V}{\partial x_{k}} d P-\frac{\partial S}{\partial x_{k}} d T+\sum_{m=1}^{n-1} \frac{\partial^{2} G}{\partial x_{k} \partial x_{m}} d x_{m}^{-}
\end{gathered}
$$

Es folgt:

$$
\begin{gathered}
\left\{V^{\prime}-V^{\prime \prime}-\sum_{k=1}^{n-1}\left(x_{k}^{\prime}-x_{k}^{\prime \prime}\right) \frac{\partial V}{\partial x_{k}}\right\} d P \\
-\left\{S^{\prime}-S^{\prime \prime}-\sum_{k=1}^{n-1}\left(x_{k}^{\prime}-x_{k}^{\prime \prime}\right) \frac{\partial S}{\partial x_{k}}\right\} d T^{(22)} \\
=\sum_{k=1}^{n-1} \sum_{m=1}^{n-1}\left(x_{k}^{\prime}-x_{k}^{\prime \prime}\right) \frac{\partial^{2} G}{\partial x_{k} \partial x_{m}} d x_{m}
\end{gathered}
$$

$\frac{\partial V}{\partial x_{k}}, \frac{\partial S}{\partial x_{k}}, \frac{\partial^{2} G}{\partial x_{k} \partial x_{m}}$ und $d x_{m}$ beziehen sich wahlweise auf eine der beiden Phasen. Diese von Storonkin ${ }^{8}$ angegebene Koexistenzgleichung für Systeme mit beliebig vielen Komponenten hat für Zweiphasengleichgewichte große Bedeutung. Sie wurde in einer früheren Mitteilung ${ }^{9}$ bei der Untersuchung von Verdampfungsgleichgewichten bereits diskutiert. An dieser Stelle soll uns nur eines interessieren: Sind die in geschweiften Klammern stehenden Ausdrücke für alle Werte von $x_{k}$ endlich? Stor onk in ${ }^{8}$ hat diese Frage ebenfalls beantwortet. Wir wollen aber einen anderen Weg gehen, der zu besonders übersichtlichen Resultaten führt und außerdem in gewissen Fällen Vorzeichenaussagen gestattet. Es wird an die vorangehenden Ausführungen über par-

8 A. Stor onkin, Acta physicochim. URSS 13, 505 [1940].

๑ R. H a a s e, Z. Naturforschg. 2a, 492 [1947]. tielle molare Zustandsgrößen angeknüpft. Dabei genügt die Durchrechnung für einen der Summenausdrücke; da der zweite völlig analog zu behandeln ist. Wir untersuchen die Summe

$V_{0}^{\prime \prime}=V^{\prime}-V^{\prime \prime}-\sum_{k=1}^{n-1}\left(x_{k}^{\prime}-x_{k}^{\prime \prime}\right)\left(\frac{\partial V}{\partial x_{k}}\right)^{\prime \prime}$.

Wenn wir in den Gln. (2a), (10), (11) als Zustandsfunktion das Volumen einführen ( $V=$ Molvolumen der Mischung, $v_{i}=$ partielles Molvolumen), so erhalten wir:

$$
\begin{aligned}
V & =\sum_{k=1}^{n} x_{k} v_{k}, \\
\frac{\partial V}{\partial x_{k}} & =v_{k}-v_{n}, \\
v_{n} & =V-\sum_{k=1}^{n-1} x_{k} \frac{\partial V}{\partial x_{k}} .
\end{aligned}
$$

Wir substituieren in (23):

$$
\begin{aligned}
& V^{\prime}=\sum_{k=1}^{n} x_{k}^{\prime} v_{k}^{\prime}=\sum_{k=1}^{n-1} x_{k}^{\prime} v_{k}^{\prime}+x_{n}^{\prime} v_{n}^{\prime}, \\
& V^{\prime \prime}=v_{n}^{\prime \prime}+\sum_{k=1}^{n-1} x_{k}^{\prime \prime}\left(\frac{\partial V}{\partial x_{k}}\right)^{\prime \prime}, \\
& n-1 \\
& \sum_{k=1}^{n} x_{k}^{\prime}\left(\frac{\partial V}{\partial x_{k}}\right)^{\prime \prime}=\sum_{k=1}^{n-1} x_{k}^{\prime}\left(v_{k}^{\prime \prime}-v_{n}^{\prime \prime}\right) .
\end{aligned}
$$

Daraus ergibt sich:

$$
\begin{gathered}
V_{0}^{\prime \prime}=\sum_{k=1}^{n-1} x_{k}^{\prime} v_{k}^{\prime}+x_{n}^{\prime} v_{n}^{\prime}-\sum_{k=1}^{n-1} x_{k}^{\prime}\left(v_{k}^{\prime \prime}-v_{n}^{\prime \prime}\right)-v_{n}^{\prime \prime} \\
=\sum_{k=1}^{n-1} x_{k}^{\prime}\left(v_{k}^{\prime}-v_{k}^{\prime}\right)+x_{n}^{\prime} v_{n}^{\prime}-v_{n}^{\prime \prime}\left(1-\sum_{k=1}^{n-1} x_{k}^{\prime}\right) \\
\text { Mit } x_{n}^{\prime}=1-\sum_{k=1}^{n-1} x_{k}^{\prime} \text { folgt: } \\
V_{0}^{\prime \prime}=\sum_{k=1}^{n} x_{k}^{\prime}\left(v_{k}^{\prime}-v_{k}^{\prime \prime}\right) .
\end{gathered}
$$


Der Unterschied der Partialvolumina einer Komponente in zwei Phasen ist eine endliche Größe. Speziell für das Gleichgewicht Flüssigkeit (") - Dampf (') ergibt sich für nicht allzu große Nähe eines kritischen Punktes: $v_{k}^{\prime}>v_{k}^{\prime \prime}$. Man erhält also: $V_{0}^{\prime \prime}>0$. Eine analoge Beziehung gilt für den Koeffizienten von $d T$ in Gl. (22).
Diese Ergebnisse wurden bei einer früheren Rechnung ${ }^{9}$ bereits benutzt.

Die mitgeteilten Gleichungen wurden bei der Aufstellung allgemeingültiger Beziehungen für die Verdampfungsgleichgewichte von Mehrstoffgemischen gewonnen. Hrn. Prof. J ost danke ich herzlich für das Interesse an der Arbeit.

\title{
Dampfdruck und Entmischung an hochmolekularen Lösungen
}

\author{
Von ERnst Jenckel \\ Aus dem Institut für theoretische Hüttenkunde und physikalische Chemie \\ der Technischen Hochschule Aachen \\ (Z. Naturforschg. 3 a, 290-298 [1948]; eingegangen am 19. Januar 1948)
}

\begin{abstract}
1. An den Systemen Polystyrol- $\alpha$-Picolin, Polystyrol-Toluol und Polymethacrylester$\alpha$-Picolin wurde zwischen $x_{2}^{\prime}=0$ bis 90 Grundmolprozenten der Dampfdruck bestimmt. Er weicht stark vom idealen Verhalten ab und läßt sich zwischen 0 und 70 Grundmolprozenten durch die Gleichung $\ln a_{1}=-K x^{\prime}{ }^{2}{ }^{2}$ mit $K=+1,111$ bzw. $+0,804$ bzw. $+1,176$ wiedergeben. 2. An der sich entmischenden Lösung Polystyrol-Octadecylalkohol wurde insbesondere der kritische Punkt bei verschiedenen Kettenlängen festgestellt. Die kritische Zusammensetzung entspricht einer sehr verdünnten Lösung, wie auch an einigen anderen hochmolekularen Lösungen bestätigt werden konnte, die kritische Temperatur steigt nur sehr wenig mit dem Polymerisationsgrad an. 3. Die Dampfdruckkurven lassen sich mit der Vorstellung der „regulären“ Lösung wiedergeben, die beobachteten kritischen Daten widersprechen ihr völlig. Letztere lassen sich aber vorzüglich aus den ,,irregulären“ Lösungen herleiten, nachdem für verdünnte Lösungen kürzlich von A. M ünster die Lösungsentropie berechnet werden konnte. 4. Unabhängig von jeder Theorie folgt aus den beobachteten Dampfdruckkurven die Ostwaldsche Extrapolationsformel auf unendliche Verdünnung.
\end{abstract}

$I^{\mathrm{n}}$

$m$ folgenden soll über Dampfdruck-Messungen an einigen hochmolekularen Lösungen berichtet werden, bei denen Wert auf einen möglichst ausgedehnten Konzentrationsbereich gelegt wurde. Ferner wurde an anderen Lösungen die Entmischung in zwei flüssige Phasen und insbesondere der kritische Punkt bestimmt. Beide Versuchsreihen lassen gewisse Besonderheiten erkennen, die offenbar für hochmolekulare Stoffe kennzeichnend sind. Wie die Thermodynamik lehrt, können diese Gleichgewichte aus den Energie- und Entropieänderungen bei der Herstellung der Lösung aus den reinen Komponenten hergeleitet werden. Es wird daher weiterhin untersucht, ob die Charakteristika der hochmolekularen Lösungen vornehmlich auf besondere Energie- oder aber auf Entropieänderungen zurückgeführt wer-

1 Im System Polystyrol-p-Xylol wurde, wohl durch einen geringen Wassergehalt des Lösungsmittels veranlaßt, ein Maximum des Dampfdrucks bei etwa 20 Grundmolprozenten Polymerisat gefunden. den müssen, mit dem Ergebnis, daß die letzteren ausschlaggebend sind.

\section{A. Experimentelles}

1. Dampfdruck (nach Versuchen von Leo Go 0 rden). Es wurden die Gemische Polystyrol- $\alpha$ Picolin, Polystyrol-Toluol und Polymethacrylester (Plexiglas) $-\alpha$-Picolin gemessen. Die Polymerisate und die niedrigmolekularen Lösungsmittel waren so ausgesucht, daß jeweils ein Polymerisat und ein Lösungsmittel mit ausgeprägtem und mit schwachem Dipolmoment verwandt wurden. Die Lösungsmittel waren sorgfältig getrocknet ${ }^{1}$ und fraktioniert.

Der Dampfdruck wurde in der in Abb. 1 skizzierten Anordnung gemessen. Zunächst wurden in mehrere Töpfe U genaue Mengen Polymerisat eingewogen und dann eine ungefähre Menge Lösungsmittel hinzupipettiert. In einem evakuierten, Calciumchlorid enthaltenden Exsiccator wurden die Gemische einige Tage sich selbst überlassen, damit die Lösungen homogen wurden. Unmittelbar vor Ansatz eines Meßversuches wurde dann die genaue jeweilige Lösungsmittelmenge zurückgewogen. Der Oberteil 0 wurde 